\title{
THE NORMALITY OF MACROCUBES AND HYPERBALLEANS
}

\author{
IGOR PROTASOV, KSENIA PROTASOVA
}

\begin{abstract}
For a bornology $\mathcal{B}$ on a cardinal $\kappa$, we prove that the $\mathcal{B}$-macrocube is normal if and only if $\mathcal{B}$ has a linearly ordered base. As a corollary, we get that the hyperballean of bounded subsets of an ultradiscrete ballean is not normal. These answer Question 1 from [2] and Question 14.4 from [1].
\end{abstract}

MSC: 54E05, 54E15, 05D10

Keywords: coarse structure, ballean, macrocube, hyperballeans, Ramsey ultrafilter.

\section{INTRODUCTION AND PRELIMINARIES}

Given a set $X$, a family $\mathcal{E}$ of subsets of $X \times X$ is called a coarse structure on $X$ if

- each $E \in \mathcal{E}$ contains the diagonal $\triangle_{X}:=\{(x, x): x \in X\}$ of $X$;

- if $E, E^{\prime} \in \mathcal{E}$ then $E \circ E^{\prime} \in \mathcal{E}$ and $E^{-1} \in \mathcal{E}$, where $E \circ E^{\prime}=\{(x, y): \exists z \quad((x, z) \in$ $\left.\left.E,(z, y) \in E^{\prime}\right)\right\}, E^{-1}=\{(y, x):(x, y) \in E\}$

- if $E \in \mathcal{E}$ and $\triangle_{X} \subseteq E^{\prime} \subseteq E$ then $E^{\prime} \in \mathcal{E}$.

Elements $E \in \mathcal{E}$ of the coarse structure are called entourages on $X$

For $x \in X$ and $E \in \mathcal{E}$ the set $E[x]:=\{y \in X:(x, y) \in \mathcal{E}\}$ is called the ball of radius $E$ centered at $x$. Since $E=\bigcup_{x \in X}\{x\} \times E[x]$, the entourage $E$ is uniquely determined by the family of balls $\{E[x]: x \in X\}$. A subfamily $\mathcal{E}^{\prime} \subseteq \mathcal{E}$ is called a base of the coarse structure $\mathcal{E}$ if each set $E \in \mathcal{E}$ is contained in some $E^{\prime} \in \mathcal{E}$.

The pair $(X, \mathcal{E})$ is called a coarse space [10] or a ballean [7, [9].

In this paper, all balleans under consideration are supposed to be connected: for any $x, y \in X$, there is $E \in \mathcal{E}$ such $y \in E[x]$. A subset $Y \subseteq X$ is called bounded if $Y=E[x]$ for some $E \in \mathcal{E}$, and $x \in X$. The family $\mathcal{B}_{X}$ of all bounded subsets of $X$ is a bornology on $X$. We recall that a family $\mathcal{B}$ of subsets of a set $X$ is a bornology if $\mathcal{B}$ contains the family $[X]^{<\omega}$ of all finite subsets of $X$ and $\mathcal{B}$ is closed under finite unions and taking subsets. A bornology $\mathcal{B}$ on a set $X$ is called unbounded if $X \notin \mathcal{B}$. A subfamily $\mathcal{B}^{\prime}$ of $\mathcal{B}$ is called a base for $\mathcal{B}$ if, for each $B \in \mathcal{B}$, there exists $B^{\prime} \in \mathcal{B}^{\prime}$ such that $B \subseteq B^{\prime}$.

Each subset $Y \subseteq X$ defines a subbalean $\left(Y,\left.\mathcal{E}\right|_{Y}\right)$ of $(X, \mathcal{E})$, where $\left.\mathcal{E}\right|_{Y}=\{E \cap(Y \times Y)$ : $E \in \mathcal{E}\}$. A subbalean $\left(Y,\left.\mathcal{E}\right|_{Y}\right)$ is called large if there exists $E \in \mathcal{E}$ such that $X=E[Y]$, where $E[Y]=\bigcup_{y \in Y} E[y]$.

Let $(X, \mathcal{E}),\left(X^{\prime}, \mathcal{E}^{\prime}\right)$ be balleans. A mapping $f: X \rightarrow X^{\prime}$ is called macrouniform if for every $E \in \mathcal{E}$ there exists $E^{\prime} \in \mathcal{E}$ such that $f(E(x)) \subseteq E^{\prime}(f(x))$ for each $x \in X$. If $f$ is a bijection such that $f$ and $f^{-1}$ are macrouniform, then $f$ is called an asymorphism. If $(X, \mathcal{E})$ and $\left(X^{\prime}, \mathcal{E}^{\prime}\right)$ contains large asymorphic subballeans, then they are called coarsely equivalent. 
Every metric $d$ on a set $X$ defines the coarse structure $\mathcal{E}_{d}$ on $X$ with the base $\{\{(x, y)$ : $d(x, y)<n\}: n \in \mathbb{N}\}$. A ballean $(X, \mathcal{E})$ is called metrizable if there is a metric $d$ on such that $\mathcal{E}=\mathcal{E}_{d}$.

Theorem 1.1. ([9, Theorem 2.1.1]) A ballean $(X, \mathcal{E})$ is metrizable if and only if $\mathcal{E}$ has a countable base.

Let $(X, \mathcal{E})$ be a ballean. A subset $U \subseteq X$ is called an asymptotic neighbourhood of a subset $Y \subseteq X$ if for every $E \in \mathcal{E}$ the set $E[Y] \backslash U$ is bounded.

Two subset $Y, Z$ of $X$ are called asymptotically disjoint (separated) if for every $E \in \mathcal{E}$ the intersection $E[Y] \cap E[Z]$ is bounded ( $Y$ and $Z$ have disjoint asymptotic neighbourhoods). We say that $Y, Z$ are linked if $Y, Z$ are not asymptotically disjoint.

A ballean $(X, \mathcal{E})$ is called normal [6] if any two asymptotically disjoint subsets of $X$ are asymptotically separated. Every ballean $(X, \mathcal{E})$ with linearly ordered base of $\mathcal{E}$ is normal. In particular, every metrizable ballean is normal, see [6, Proposition 1.1].

A function $f: X \rightarrow \mathbb{R}$ is called slowly oscillating if for any $E \in \mathcal{E}$ and $\varepsilon>0$, there exists a bounded subset $B$ of $X$ such that diam $f(E[x])<\varepsilon$ for each $x \in X \backslash B$.

Theorem 1.2. ([6, Theorem 2.1.]) A ballean $(X, \mathcal{E})$ is normal if and only if for any two disjoint asymptotically disjoint subsets $Y, Z$ of $X$ there exists a slowly oscillating function $f: X \rightarrow[0,1]$ such that $\left.f\right|_{Y} \equiv 0$ and $\left.f\right|_{Z} \equiv 1$.

Theorem 1.3. ([1, Theorem 1.4 .]) If the product $X \times Y$ of two unbounded balleans $X, Y$ is normal then the bornology $B_{X \times Y}$ has a linearly ordered base.

\section{MaCROCUBES AND HYPERBALLEANS}

Let $\mathcal{B}$ be a bornology on an infinite cardinal $\kappa$ such that $\kappa \notin \mathcal{B},\{0,1\}^{\mathcal{B}}=\left\{\left(x_{\alpha}\right)_{\alpha<\kappa}\right) \in$ $\{0,1\}^{\kappa}:\left\{\alpha: x_{\alpha}=1\right\} \in \mathcal{B}$. We take the family

$$
\left\{\left\{\left(\left(x_{\alpha}\right)_{\alpha<\kappa}, \quad\left(y_{\alpha}\right)_{\alpha<\kappa}\right) \in\{0,1\}^{\mathcal{B}} \times\{0,1\}^{\mathcal{B}}: x_{\alpha}=y_{\alpha}, \alpha \in \kappa \backslash B\right\}: B \in \mathcal{B}\right\}
$$

as a base of the coarse structure on $\{0,1\}^{\mathcal{B}}$. The obtained ballean is called the $\mathcal{B}$ macrocube [2]. If $\kappa=\omega$ and $\mathcal{B}=[\omega]^{<\omega}$ then we get the well-known Cantor macrocube whose coarse characterization was given in $[3$.

Given a ballean $(X, \mathcal{E})$ the hyperballean $(X, \mathcal{E})^{b}$ is a ballean on $\mathcal{B}_{(X, \mathcal{E})}$ endowed with a coarse structure with the base $\left\{E^{b}: E \in \mathcal{E}\right\}, E^{b}[Y]=\{Z: Y \subseteq E[Z], Z \subseteq E[Y]\}$, see [4, 8].

Every bornology $\mathcal{B}$ on a cardinal $\kappa$ defines the discrete ballean $\left(\kappa, \mathcal{E}_{\mathcal{B}}\right), \mathcal{E}_{\mathcal{B}}$ has the base $\left\{E_{\mathcal{B}}: B \in \mathcal{B}\right\}, E_{\mathcal{B}}[x]=B$ if $x \in B$ and $E_{\mathcal{B}}[x]=\{x\}$ if $x \in \kappa \backslash B$. If the set $\{\kappa \backslash B: B \in \mathcal{B}\}$ is an ultrafilter, then $\left(\kappa, \mathcal{E}_{\mathcal{B}}\right)$ is called ultradiscrete. We denote by $[\kappa]^{\mathcal{B}}$ the hyperballean $\left(\kappa, \mathcal{E}_{\mathcal{B}}\right)^{b}$.

Proposition 2.1. Let $\kappa$ be a cardinal, $\mathcal{B})$ be a bornology on $\kappa, K_{0}=\left\{A \in[\kappa]^{\mathcal{B}}\right.$ : $0 \in A$. Then the characteristic function $f:[\kappa]^{\mathcal{B}} \longrightarrow\{0,1\}^{\mathcal{B}}$ is macro-uniform and the restriction of $f$ to $K_{0}$ is an asymorphism between $K_{0}$ and $f\left(K_{0}\right)$. 
Proposition 2.2. For any cardinal $\kappa$ and bornology $\mathcal{B}$ on $\kappa$, the balleans $\{0,1\}^{\mathcal{B}}$ and $[\kappa]^{\mathcal{B}}$ are not coarsely equivalent.

Proof. Following [3], we say that a ballean $(X, \mathcal{E}$ has asymptotically isolated balls if, for any bounded subset $B$ and $E \in \mathcal{E}$ there exists $x \in X \backslash B$ such that $E^{\prime}[x] \backslash E[x]=\emptyset$. If $\left(X^{\prime}, \mathcal{E}^{\prime}\right)$ is coarsely equivalent to $(X, \mathcal{E})$ then $\left(X^{\prime}, \mathcal{E}^{\prime}\right)$ has asymptotically isolated balls.

We observe $\{0,1\}^{\mathcal{B}}$ does not have asymptotically isolated balls, but the subballean $[\kappa]_{1}^{\mathcal{B}}=\{A \subset \kappa:|A|=1\}$ of $[\kappa]^{\mathcal{B}}$ has asymptotically isolated balls. Since $[\kappa]_{1}^{\mathcal{B}}$ and $[\kappa]^{\mathcal{B}} \backslash[\kappa]_{1}^{\mathcal{B}}$ are asymptotically disjoint, we see that $[\kappa]^{\mathcal{B}}$ has asymptotically isolated balls.

Proposition 2.3. Let $\mathcal{B}$ be a bornology on a cardinal $\kappa$ such that $\{\kappa \backslash B: B \in \mathcal{B}\}$ is not an ultrafilter. Then $[\kappa]^{\mathcal{B}}$ is normal if and only if $\mathcal{B}$ has a linearly ordered base.

Proof. If $\mathcal{B}$ has a linearly ordered base then $[\kappa]^{\mathcal{B}}$ is normal by [6, Proposition 1.1].

We assume that $[\kappa]^{\mathcal{B}}$ is normal, partition $\kappa$ in two unbounded subsets $K_{1}, K_{2}$ and put $\mathcal{B}_{1}=\left.\mathcal{B}\right|_{K_{1}}, \mathcal{B}_{2}=\left.\mathcal{B}\right|_{K_{2}}$. Then $\{0,1\}^{\mathcal{B}}$ is asymorphic to $\{0,1\}^{\mathcal{B}_{1}} \times\{0,1\}^{\mathcal{B}_{2}}$. By Theorem $1.3, \mathcal{B}$ has a linearly ordered base.

\section{Some Algebra on Ultrafilters}

Let $G$ be an Abelian group. We endow $G$ with the discrete topology and identify the Stone- $C$ ech compactification $\beta G$ of $G$ with the family of all ultrafilters on $G$.

For $A \subseteq G$, we denote $\bar{A}=\{\mathcal{U} \in \beta G: A \in \mathcal{U}\}$.

Then the family $\{\bar{A}: A \subseteq G\}$ forms a base for the topology on $\beta G$. Every mapping $f: G \longrightarrow[0,1]$ can be extended to the continuous mapping $f^{\beta}: \beta G \longrightarrow[0,1]$.

Following [5], we extend the addition + on $G$ onto $\beta G$ by the following rule: for $\mathcal{U}$, $\mathcal{V} \in \beta G$ we take $U \in \mathcal{U}$ and, for each $x \in U$, pick $V_{x} \in \mathcal{U}$. Then $\bigcup_{x \in U}\left(x+V_{x}\right) \in \mathcal{U}+\mathcal{V}$, and the family of all these subsets forms a base of the ultrafilter $\mathcal{U}+\mathcal{V}$.

\section{Results}

Theorem 4.1. Let $\mathcal{B}$ be a bornology on a cardinal $\kappa$. The $\mathcal{B}$-macrocube $X$ is normal if and only if $\mathcal{B}$ has a linearly ordered base.

Poof. In light of Proposition 2.3, it suffices to assume that $\{\kappa \backslash B: B \in \mathcal{B}\}$ is an ultrafilter and show that $X$ is not normal.

We consider $X$ as a group with pointwise addition $\bmod 2$. For $A \subseteq \kappa$ and $\alpha<\kappa, \chi_{A}$ denotes the characteristic function of $A,[0 . \alpha]=\{\gamma<\kappa: \gamma \leq \alpha\}$. Replacing $\kappa$ to some cardinal $\kappa^{\prime} \leq \kappa$, we may suppose that $[0, \alpha] \in \mathcal{B}$ for each $\alpha<\kappa$.

We consider two subsets $Y, Z$ of $X$ defined by

$$
\begin{aligned}
& Y=\left\{y_{\alpha}: \alpha<\kappa\right\}, y_{\alpha}=\chi_{\{\alpha\}}, \\
& Z=\left\{z_{\alpha}: \alpha<\kappa\right\}, z_{\alpha}=\chi_{[0, \alpha]},
\end{aligned}
$$


and show that $Y, Z$ are asymptotically disjoint. We take an arbitrary $B \in \mathcal{B}$, denote by $H$ the subgroup of $X$ generated by $\left\{y_{\alpha}: \alpha \in B\right\}$ and take the minimal $\gamma<\kappa$ such that $\gamma \in \kappa \backslash B$. Then $\left(y_{\beta}+H\right) \cap Z=\emptyset$ as soon as $\beta \in \kappa \backslash B, \beta>\gamma$.

We suppose that $X$ is normal and use Theorem 1.2 to choose a slowly oscillating function $f: X \longrightarrow[0,1]$ such that $\left.f\right|_{Z} \equiv 1,\left.f\right|_{Y} \equiv 0$, and denote by $\mathcal{U}$ and $\mathcal{V}$ ultrafilters on $X$ with the bases

$$
\left\{\left\{y_{\alpha}: \alpha \in \kappa \backslash B\right\}: B \in \mathcal{B}\right\},\left\{\left\{z_{\alpha}: \alpha \in \kappa \backslash B\right\}: B \in \mathcal{B}\right\}
$$

Let $f^{\beta}(\mathcal{V}+\mathcal{U})=r, r \in[0.1]$. We take an arbitrary $W \in \mathcal{V}+\mathcal{U}$ and pick $C \in \mathcal{B}$ and $D_{\alpha} \in \mathcal{B}, \alpha \in C$ such that

$$
\bigcup\left\{z_{\alpha}+y_{\beta}: \alpha \in \kappa \backslash C, \beta \in \kappa \backslash D_{\alpha}\right\} \subseteq W .
$$

Then we construct inductively two mappings $\psi: \kappa \backslash C \longrightarrow \kappa, \phi: \kappa \backslash C \longrightarrow \kappa$ such that $\psi(\alpha) \in \kappa \backslash D_{\alpha}, \phi(\alpha) \in \kappa \backslash D_{\alpha}$ and $\psi(\kappa \backslash C) \cap \phi(\kappa \backslash C)=\emptyset$. Since $\{\kappa \backslash B: B \in \mathcal{B}\}$ is an ultrafilter, either $\psi(\kappa \backslash C) \in \mathcal{B}$ or $\phi(\kappa \backslash C) \in \mathcal{B}$. We assume that $\psi(\kappa \backslash C) \in \mathcal{B}$ and denote by $H$ the subgroup of $X$ generated by $\left\{y_{\alpha}: \alpha \in \psi(\kappa \backslash C)\right\}$.

Then $z_{\alpha} \in W+H$ for each $\alpha \in \kappa \backslash C$. Hence, $W$ and $Z$ are linked. Since $f$ is slowly oscillating, we conclude that $r=1$.

On the other hand, for $\alpha \in \kappa \backslash C, z_{\alpha}+\left\{y_{\beta}: \beta \in \kappa \backslash D_{\alpha}\right\} \subseteq W$. It follows that $W$ and $Y$ are linked. Since $f$ is slowly oscillating, we get $r=0$ contradicting above paragraph.

Corollary 4.2. Let $\mathcal{B}$ be a bornology on a cardinal $\kappa$ such that $\{\kappa \backslash B: B \in \mathcal{B}\}$ is an ultrafilter. Then the hyperballean $[\kappa]^{\mathcal{B}}$ is not normal.

Poof. Since a subballean of a normal ballean is normal, to apply Theorem 4.1, we use Proposition 2.1.

Theorem 4.1 answers Question 1 from [2], Corollary 4.2 answers Question 14.4 from [1].

For a bornology $\mathcal{B}$ on a cardinal $\kappa$, the subballean of all characteristic functions of finite subsets of $\kappa$ of the ballean $\{0,1\}^{\mathcal{B}}$ is called the finitary $\mathcal{B}$-macrocube. It follows from the proof of Theorem 4.1 that the finitary $\mathcal{B}$-macrocube on $\omega$ is not normal provided that $\{\omega \backslash B: B \in \mathcal{B}\}$ is ultrafilter.

Question 4.3. Let $\mathcal{B}$ be a bornology on a cardinal $\kappa$ such that $\{\kappa \backslash B: B \in \mathcal{B}\}$ is an ultrafilter. Is it true that the finitary $\mathcal{B}$-macrocube is not normal?

For $n \in \omega, n>0$ and a bornology $\mathcal{B}$ on $\kappa$, we denote $[\kappa]_{n}^{\mathcal{B}}=\left\{A \in[\kappa]^{\mathcal{B}}:|A| \leq n\right\}$, $\{0,1\}_{n}^{\mathcal{B}}=\left\{\left(x_{\alpha}\right)_{\alpha<\kappa}:\left|\left\{\alpha: x_{\alpha}=1\right\}\right| \leq n\right\}$. If $\{\kappa \backslash B: B \in \mathcal{B}\}$ is an ultrafilter then $[\kappa]_{n}^{\mathcal{B}}$ is normal [1, Theorem 1.13].

A ballean $(X, \mathcal{E})$ is called ultranormal if any two unbounded subsets of $X$ are linked. 
Theorem 4.4. Let $\mathcal{B}$ be a bornology on $\kappa$ such that $\{\kappa \backslash B: B \in \mathcal{B}\}$ is an ultrafilter. Then the ballean $\{0,1\}_{n}^{\mathcal{B}}$ is ultranormal.

Proof. We consider $\{0,1\}_{1}^{\mathcal{B}}$ as a subballean of $\{0,1\}_{n}^{\mathcal{B}}$. Since $\{\kappa \backslash B: B \in \mathcal{B}\}$ is an ultrafilter, it suffices to show that $\mathcal{A},\{0,1\}_{1}^{\mathcal{B}}$ are linked for any unbounded subset $\mathcal{A}$ of $\{0,1\}_{n}^{\mathcal{B}}$.

Applying $n$-times Lemma 7.1 from [1, we pick $P \subseteq \kappa$ such that $\kappa \backslash P \in \mathcal{B}$ and $\mathcal{A}^{\prime}=\{A \in \mathcal{A}:|A \cap P|=1\}$ is unbounded. We put $H=\left\{\left(x_{\alpha}\right)_{\alpha<\kappa}: x_{\alpha}=0\right.$ for each $\alpha \in P\}$. Then $(x+H) \cap \mathcal{A}^{\prime} \neq \emptyset$ for each $x \in P$. Hence, $\mathcal{A}^{\prime}$ and $\{0,1\}_{1}^{\mathcal{B}}$ are linked.

\section{REFERENCES}

[1] T. Banakh, I. Protasov, The normality and bounded growth of balleans, https://arxiv.org/abs/1810.07979.

[2] T. Banakh, I. Protasov, Constructing balleans, J. Math. Sciences, 241 (2019), 16-26.

[3] T. Banakh, I. Zarichnyi, Characterizing the Cantor bi-cube in asymptotic categories, Groups Geom. Dyn. 5: 4 (2011), 691-728.

[4] D. Dikranjan, I. Protasov, K. Protasova, N. Zava, Balleans, hyperballeans and ideals, Appl. Gen. Topology 2: 4 (2019), 431-447.

[5] N. Hindman, D. Strauss, Algebra in the Stone-Č ech Compactification, de Gructer, Berlin, New York, 1998.

[6] I. Protasov, Normal ball structures, Math. Stud. 20 (2003), 3-16.

[7] I. Protasov, T. Banakh, Ball Structures and Colorings of Groups and Graphs, Math. Stud. Monogr. Ser., Vol. 11, VNTL, Lviv, 2003.

[8] I. Protasov, K. Protasova, On hyperballeans of bounded geometry, Europ. J. Math. 4 (2018), 15151520.

[9] I. Protasov, M. Zarichnyi, General Asymptology, Math. Stud. Monogr. Ser., Vol. 12, VNTL, Lviv, 2007, pp. 219.

[10] J. Roe, Lectures on Coarse Geometry, Univ. Lecture Ser., vol. 31, American Mathematical Society, Providence RI, 2003.

I.Protasov: Faculty of Computer Science and Cybernetics, Kyiv University, Academic Glushkov PR. 4D, 03680 KYIV, UKRAINE

E-mail address: i.v.protasov@gmail.com

K.Protasova: Faculty of Computer Science and Cybernetics, Kyiv University, Academic Glushkov PR. 4D, 03680 KYIV, UKRAINE

E-mail address: k.d.ushakova@gmail.com 